\title{
Field Survey Considering Distribution of Fire Location Part 2 Consideration for Two Types of Space Area
}

\author{
Jun Kitahori $^{1}$, Yoshifumi Ohmiya ${ }^{1}$, Norichika Kakae ${ }^{2}$, Ken Matsuyama ${ }^{1}$ \\ and Kazunori Harada ${ }^{2}$ \\ ${ }^{\text {I}}$ Tokyo University of Science \\ ${ }^{2}$ Kyoto University
}

Keywords : Flammable Materials, Field Survey, Local Fire, Heat Release Rate, Exposed Surface Area, Offset Distance

\section{INTRODUCTION}

As a sequel to Part 1, Part 2 of this survey will present the considerations given to the survey results presented in Part 1. As mentioned in Part 1, the lobby and hallway are referred as "walkway areas" and the lounge and meeting room as "room areas".

\section{CONSIDERATIONS}

\subsection{Load of flammable material}

As shown in Figure 1, the equivalent load of flammable materials (total heat release rate) and the valid load of flammable materials (valid heat release rate) tends to be larger as the floor area increases. In the walkway area, as the floor area increases from approximately 80 to $100 \mathrm{~m}^{2}$, the equivalent and the valid loads of the flammable materials start to increase. This is probably because of the increased amount of flammable material in more spacious floor areas that can be utilized for purposes other than walkways, whereas smaller spaces will only be used as walkways.

When comparing the absolute values of the flammable material loads between the room space and walkway space, the values of the flammable material load with respect to the floor area is higher in the room space than walkway space.
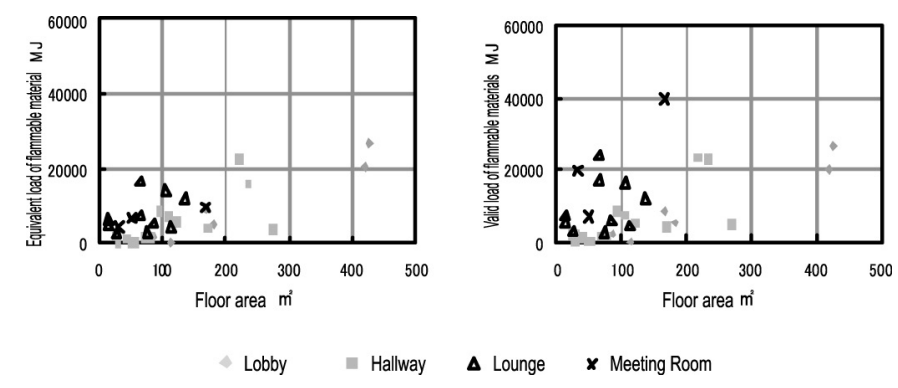

Figure 1 Load of flammable material and floor area 


\subsection{Concentration of flammable materials}

Table 1 describes the data obtained from this survey with respect to the concentration of flammable materials. Figure 2 illustrates the relation between concentration of flammable materials and floor area. According to the results, in the room space, especially in the lounge, the concentration of flammable materials tends to decrease as the floor area increases. On the other hand, in the walkway area, the concentration of flammable materials tends to be constant or slightly increases as the floor area increases. In the room space, regardless of the size of the space, the concentration of flammable materials becomes higher in smaller rooms depending on the furniture and equipment are carried into the room to facilitate its use. On the other hand, in the walkway areas, especially in the hallway, since the flammable materials are placed along with walls, the flammable material per unit length of the hallway becomes almost constant. It is presumed that the concentration of flammable materials will have the same patterns as above.

Table 1 Survey results of the concentration of flammable materials

\begin{tabular}{|c|c|c|c|c|c|}
\hline \multicolumn{5}{|c|}{ Equivalent concentration of flammable materials MJ $/ \mathrm{m}^{2}$} \\
\hline Space type & Use of room & Range & $\mu_{w}$ & $\sigma_{w}$ & Building Standards Law \\
\hline \multirow{3}{*}{ Walkway } & Hallway & 0.2 to 104.0 & 38.6 & 32.8 & 32 \\
\cline { 2 - 6 } & Lobby & 0.8 to 82.4 & 35.1 & 23.2 & 80 \\
\hline \multirow{2}{*}{ Room } & Lounge & 36.7 to 481.5 & 156.6 & 148.6 & 400 \\
\cline { 2 - 6 } & Meeting Room & 57.2 to 129.3 & 104.4 & 40.9 & 160 \\
\hline \multirow{6}{*}{ Walkway } & Valid concentration of flammable materials $\mathrm{MJ} / \mathrm{m}^{2}$ \\
\hline \multirow{2}{*}{ Room } & Lobby & 0.2 to 108.1 & 43.2 & 37.2 & 32 \\
\cline { 2 - 6 } & Lounge & 36.8 to 62.4 & 35.6 & 22.8 & 80 \\
\cline { 2 - 6 } & Meeting Room 547.3 & 134.5 to 624.3 & 332.5 & 258.0 & 400 \\
\hline
\end{tabular}
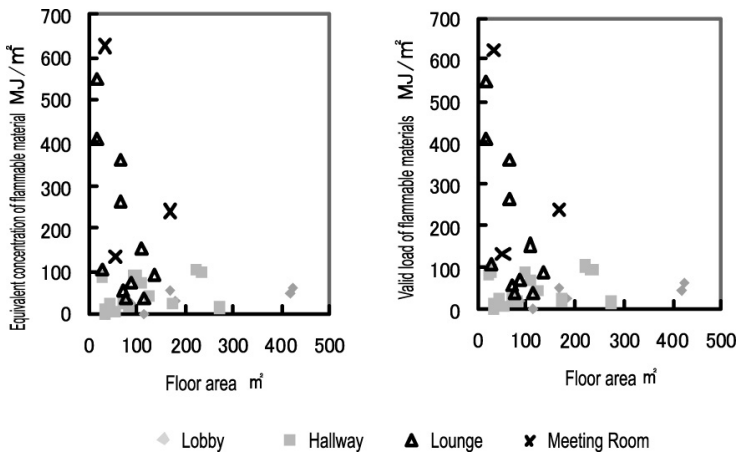

Figure 2 Concentration of flammable material and floor area 


\subsection{Surface area coefficient}

Figure 3 shows the relation between concentration of flammable materials and surface area coefficient. However, the surface area coefficient is the value of which the exposed surface area was divided by the equivalent load of flammable materials, and the valid surface area coefficient is the value of which the valid exposed surface area was divided by the valid load of flammable materials.

According to the data obtained in a past survey that was conducted with respect to the flammable materials in an office, the relation between equivalent concentration of the flammable materials and surface area coefficient is proportional to the square of $-2 / 3$. When comparing the result of this experiment with the expression proposed for an office-use room in the past survey, the value of the surface area coefficient tends to be lower in the walkway space of this survey and higher in the room space of this survey. Also, the declining rate of the surface area coefficient, which was accompanied by an increase in the equivalent concentration of flammable materials, tends to be smaller than that proposed in the past survey.

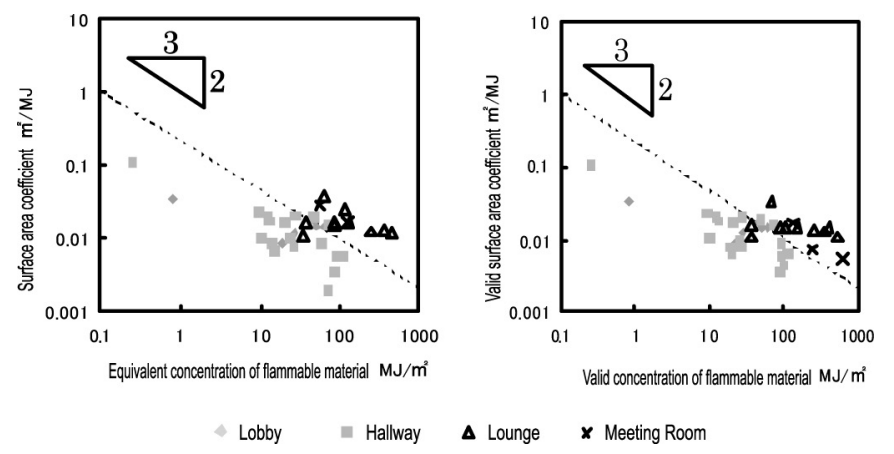

Figure 3 Surface area coefficient and concentration of flammable materials

\subsection{Exposed Surface Area}

Figure 4 shows the relation of exposed surface area and floor area. In Figure 4, the survey results of the administration office (dashed line) and the engineering office (solid line) are also shown. According to this figure, the proportion of the exposed surface area to the floor area tends to be greater in room space than in walkway space. The proportion of the exposed surface area to the floor area tends to be higher in room space than in walkway space. The value is the same or slightly higher in the engineering room, and the value of the walkway space is lower than that of the administrative office. 

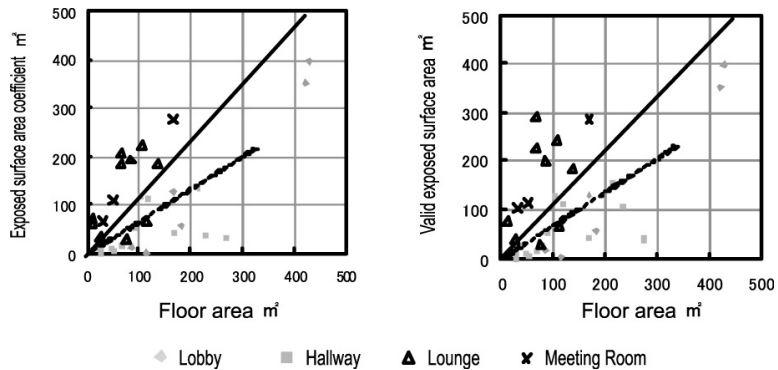

Figure 4 Exposed surface area and floor area

\subsection{Offset distance}

As shown in Figure 5, the shortest distance between the flammable materials and walls was defined as "offset distance", and it was calculated for all materials in this survey. However, the flammable materials placed on or below furniture and equipment, materials stored on the storage cases, materials on the walls, and materials on the floor were ignored.

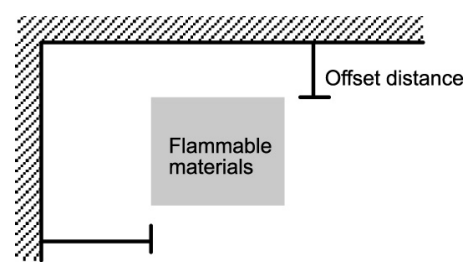

Figure 5 Definition of the offset distance

Figure 6 is a contour diagram illustrating the occupancy condition of the flammable materials when point $\mathrm{A}$ is the origin. The percentages occupied by the flammable materials are described with the contour drawn at 5\% intervals. According to Figure 6, in every room, the closer the wall, the more flammable materials are placed there, and when moving away from the wall, the height of the flammable materials becomes shorter. In per space aspect, the flammable materials are concentrated on the side of walls in the hallway. In the room space such as the lounge and meeting room, the flammable materials tend to be placed by keeping a certain distance from the walls, like an island, instead of being placing directly against the walls. 

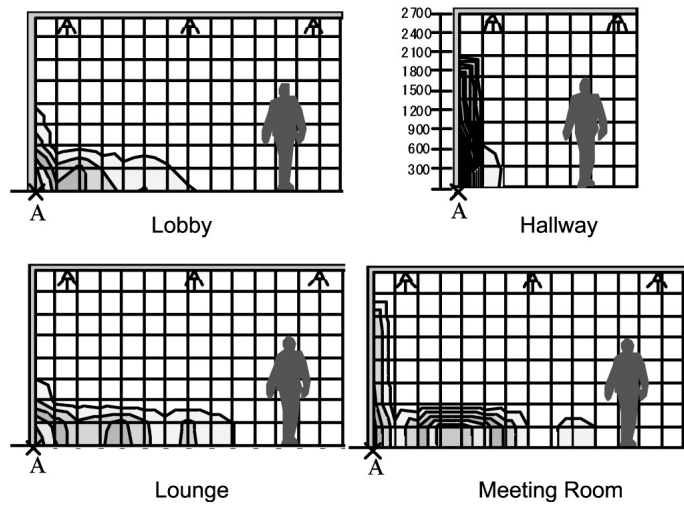

Figure 6 Contour diagram of flammable material occupancy

\section{CONCLUSIONS}

In this report, by using the survey results obtained by the method described in Part 1 , a discussion was presented with respect to the quantity and layout of flammable materials by categorizing the space by its use (walkway area or room area). The results indicated that the quantity and the layout of the flammable materials vary depending on the use of space. In further research, more surveys and experiments will be conducted with respect to a space in which a "local fire" can be anticipated.

\section{NOTE}

The content of this report is a part of the content used for the joint working groups (WG), which were undertaken by WG of the Fire-Resistance Design for Localized Fires and WG of the Fire Property under the AIJ Prevention Control Committee and the Fire Safety Committee. The members of those committees and observers are as follows (random order): As of 2004: Kazunori Harada (Kyoto Univ., PM of the fire-resistance design for localized fire WG), Yoshifumi Ohmiya (Tokyo Univ. of Science, PM of the fire model WG), Toshihiko Abe (Sanyu Civil Engineering), Ayako Ito (Tokyo Fire Dept., Research Assoc. of the Building Research Institute), Masafumi Sato (Building Center of Japan), Keiichi Suzuki and Hiroaki Notake (Shimizu Corp, TRI), Wataru Takahashi (ING), Naohiro Takeichi and Tsutomu Nagaoka (Takenaka Corp), Tsuneto Tsuchihashi and Akiko Nakamichi (General Building Research Corporation of Japan), Ichiro Nakaya (Japan Testing Center for Construction Materials), Akiko Natori (Non-Life Insurance Rating Organization of Japan), Masahiko Honma (Obayashi Corp), Ken Matsuyama (Tokyo Univ of Science), Shinji Yagi (AXS Sato Inc), Takashi Wakamatsu (Nihon Bousai Inst. Ltd) 